\title{
KMAX AND STABLE RANK OF ENVELOPING ALGEBRAS
}

\author{
GEORGE TINTERA
}

(Communicated by Maurice Auslander)

\begin{abstract}
We calculate upper bounds for the stable rank of enveloping algebras of all finite-dimensional nonabelian nilpotent and some solvable Lie algebras using Kmax and Stafford's generalization of Bass's Stable Range Theorem. We find that the stable rank of these algebras is less than their commutative counterparts, polynomial rings.
\end{abstract}

In this paper we consider the stable rank and Kmax of enveloping algebras of many nonabelian solvable Lie algebras, including all nonabelian nilpotent Lie algebras over a field of characteristic zero. The principal result (Theorem) is that the stable rank of an enveloping algebra of a nonabelian Lie algebra is usually less than the stable rank of the enveloping algebra of the abelian Lie algebra of the same dimension. We now give the definition of stable rank of a ring, state that result, and outline some applications.

Stable rank was first used by Bass in [B] under the name stable range to determine the stabilization of the first $\mathrm{K}$-theoretic group $\mathrm{K}_{1}$. We give a contemporary definition for the stable rank of a ring $R$ with identity following [MR, §6.7]. The stable rank of $R$, abbreviated $\operatorname{sr}(R)$, is the least integer $n$, such that for every $a_{1}, \ldots, a_{n+1} \in R$ with $R=a_{1} R+\cdots+a_{n+1} R$ there exist $g_{1}, \ldots, g_{n} \in R$ such that $R=\left(a_{1}+a_{n+1} g_{1}\right) R+\cdots+\left(a_{n}+a_{n+1} g_{n}\right) R$.

The stable rank of commutative polynomial rings over fields has been determined by Suslin in [Su]. If $R$ is such a polynomial ring in $n$ indeterminates, then $R$ is isomorphic to the enveloping algebra $U(\mathbf{g})$ of the abelian Lie algebra $\mathbf{g}$ of dimension $n$. Then $\operatorname{sr}(U(\mathbf{g})) \leq n+1$, with equality, if the field has transcendence degree over the field of rational numbers exceeding $n$. For enveloping algebras of many nonabelian Lie algebras, which we can think of as noncommutative polynomial rings, the picture is quite different. Let $\mathbf{g}$ be a nonabelian nilpotent Lie algebra. Then $\operatorname{Kmax}(U(\mathbf{g}))<n(\S 2$, Lemma 3). Our theorem now follows from Stafford's generalization of the Bass Stable Range Theorem in [St2]: If $R$ is a right noetherian ring, then $\operatorname{sr}(R) \leq \mathrm{Kmax}(R)+1$.

Theorem. Let $\mathbf{g}$ be a nonabelian nilpotent Lie algebra of dimension $n$. Then $\operatorname{sr}(U(\mathbf{g})) \leq n$.

Received by the editors August 26, 1991 and, in revised form, March 9, 1992; a preliminary report on the contents of this paper was presented to the Warwick Algebra Symposium, July 18, 1991.

1991 Mathematics Subject Classification. Primary 19B10, 17C25; Secondary 16A05. 
We will call a nonabelian nilpotent Lie algebra $\mathbf{g}$ over a field $k$ a Heisenberg Lie algebra if $\mathbf{g} / k X$ is an abelian Lie algebra for some $X \in \mathbf{g}$. To determine a lower bound for the stable rank of an $n$-dimensional Heisenberg Lie algebra over some fields, we can apply the above-mentioned result of [Su] to the $(n-1)$ dimensional Lie algebra $\mathbf{g} / k X$. So $\operatorname{sr}(U(\mathbf{g} / k X))=n$ over appropriate fields. Since stable rank does not increase when passing to a factor ring (cf. [B]), we have the following result.

Corollary 1. Let $\mathbf{g}$ be a Heisenberg Lie algebra of dimension $n$ over a field with transcendence degree over the field of rational numbers exceeding $n$. Then $\operatorname{sr}(U(\mathbf{g}))=n$.

The strength of the applications of stable rank increases for smaller values of the stable rank. We cite, for example, improvements of [St 1, Corollary; MR, Corollary 11.4.8(ii)] in the case $\mathbf{g}$ is a nonabelian nilpotent Lie algebra. See [B] for unexplained terminology.

Corollary 2. Let $\mathbf{g}$ be a nonabelian nilpotent Lie algebra over a field $k$ of dimension $n$. Then:

(i) For $m \geq n+1, E_{m}(U(\mathbf{g}))=\mathrm{GL}_{m}(U(\mathbf{g}))^{\prime}$, the derived subgroup, and $\mathrm{GL}_{m}(U(\mathbf{g})) / \mathrm{GL}_{m}(U(\mathbf{g}))^{\prime} \simeq k^{*}$, the multiplicative group of nonzero elements of $k$. So $\mathrm{GL}_{m}(U(\mathbf{g}))$ is finitely generated if and only if $k$ is finite.

(ii) For $m \geq n$ and $U(\mathbf{g})$-modules $X$ and $Y, X \oplus U(\mathbf{g})^{m+1} \simeq Y \oplus U(\mathbf{g})$ implies $X \oplus U(\mathbf{g})^{m} \simeq Y$.

Finally, we will use other techniques involving Ore extensions of commutative rings in $\S 3$ to get similar results for some examples of solvable Lie algebras, including the 2-dimensional nonabelian Lie algebra.

\section{KMAX}

In this section we define Kmax and develop sufficiently many properties of it in order to prove the following lemmas. The reader is referred to [St2], in which Kmax was first used, for more details.

In order to define Kmax, we must consider the deviation of posets as defined by Gabriel and Rentschler in [GR] and developed in McConnell and Robson's book [MR, §6.1]. Let $\mathscr{P}$ be a poset and $a, b$ be elements of $\mathscr{P}$. If $a \geq b$, we define the factor of $a$ by $b$ to be $\mathscr{P}_{a, b}=\{x \in \mathscr{P}: a \geq x \geq b\}$. Then $\mathscr{P}_{a, b}$ is a subposet of $\mathscr{P}$.

To define the deviation of a poset $\mathscr{P}, \operatorname{or} \operatorname{dev}(\mathscr{P})$ for short, we say that $\operatorname{dev}(\mathscr{P})=-\infty$ if $\mathscr{P}$ is discrete. For an integer $\alpha \geq 0$, we say $\operatorname{dev}(\mathscr{P})=\alpha$ if $\operatorname{dev}(\mathscr{P})$ is not less than $\alpha$, and for every decreasing chain $a_{1} \geq \cdots \geq a_{n}$ $\geq \cdots$ of elements of $\mathscr{P}, \operatorname{dev}\left(\mathscr{P}_{a_{n+1}, a_{n}}\right)<\alpha$ for all but finitely many $n$. An immediate consequence of this definition is that if $\mathscr{P}$ is a subposet of $\mathscr{Q}$, then $\operatorname{dev}(\mathscr{P}) \leq \operatorname{dev}(\mathscr{Q})$. Another fact about posets is that the deviation of a finite Cartesian product of posets is equal to the supremum of the deviation of the posets (see [MR, 6.1.14]).

Let $R$ be an associative ring with identity and $\mathscr{M}_{R}$ the set of maximal right ideals of $R$. For a proper right ideal $I$ of $R$, we write $J(I)=\bigcap\left\{M \in \mathscr{M}_{R}: I \subseteq\right.$ $M$ \}. Since the intersection of an empty collection of right ideals of $R$ is $R$, we have $J(R)=R$. Following [St2] we call a right ideal $I$ of $R$ that satisfies 
$J(I)=I$ a Jacobson right ideal. Let $\mathscr{L}(R)$ be the poset of Jacobson right ideals of $R$ with < given by set theoretic inclusion.

Now we define $\operatorname{Kmax}(R)$ to be $\operatorname{dev}(\mathscr{L}(R))$. This compares with (Gabriel and Rentschler) Krull dimension (or Kdim for short), which is to be defined to be the deviation of the poset of all right ideals of a ring. Since $\mathscr{L}(R)$ is a subposet of the poset of all right ideals of $R$, we have $\operatorname{Kmax}(R) \leq \operatorname{Kdim}(R)$ whenever the right-hand side exists. In particular, Kmax is defined for right noetherian rings, including the enveloping algebras of finite-dimensional Lie algebras.

In order to study ring extensions $R \subseteq T$, we have

Lemma 1. Let $R \subseteq T$ be a ring extension such that if $I$ is a proper right ideal of $R$, then $I T$ is a proper right ideal of $T$. Then $\operatorname{Kmax}(R) \leq \operatorname{Kmax}(T)$.

Proof. Define a mapping $\mathscr{L}(R) \rightarrow \mathscr{L}(T)$ by $I \mapsto J(I T)$. To prove the lemma, it suffices to show that the mapping is one-to-one. Suppose $I$ is any right ideal of $R$ and $M$ is a maximal right ideal of $R$ for which $M \cap I \subset I$. Then $I+M=R$. Suppose that $J((M \cap I) T)=J(I T)$. If $N$ is a right ideal of $T$ containing $M T$, then $N$ contains $(M \cap I) T$ and, by assumption, $I T$. Thus $N$ contains $R$. This contradiction shows that we must have $J((M \cap I) T) \subset J(I T)$ so that the mapping preserves proper inclusions. Thus the mapping is one-toone, proving the lemma. have

Since ring extensions $R \subseteq T$ with ${ }_{R} T$ free preserve proper right ideals, we

Corollary 3. If $R \subseteq T$ is a ring extension such that $T$ is a free left $R$-module, then $\mathrm{Kmax}(R) \leq \mathrm{K} \max (T)$.

Now we give a lemma, which is a general statement about the Jacobson right ideals in a domain. Recall that an element $z$ in a ring $R$ is said to be normal if $R z=z R$. If $z \in R$ is a normal element, denote by $R_{z}$ the localization of $R$ at powers of $z$.

Lemma 2. Let $R$ be a domain for which $\operatorname{Kmax}(R)$ is defined and let $z \in R$ be a normal element. Then $\operatorname{Kmax}(R) \leq \sup \left\{\operatorname{Kmax}(R / z R), \operatorname{Kmax}\left(R_{z}\right)\right\}$.

Proof. Let $P=z R$ and $T=R_{z}$. For $J \in \mathscr{L}(R)$, let

$$
\begin{aligned}
& J_{0}=\bigcap\left\{M \in \mathscr{M}_{R}: J+P \subseteq M\right\}, \\
& J_{1}=\bigcap\left\{M \in \mathscr{M}_{R}: J \subseteq M, P \nsubseteq M\right\} .
\end{aligned}
$$

Define a mapping $\mathscr{L}(R) \rightarrow \mathscr{L}(R / P) \times \mathscr{L}(T)$ by $J=J_{0} \cap J_{1} \mapsto\left(\overline{J_{0}}, J_{1} T\right)$, where $\bar{J}$ denotes the canonical image of $J$ in $R / P$. The lemma follows once it is clear that this mapping is well defined and one-to-one.

Clearly $\overline{J_{0}} \in \mathscr{L}(R / P)$, so we will show that $J_{1} T \in \mathscr{L}(T)$. So suppose $J_{1}=\bigcap M$, where $M$ ranges over some subset of $\mathscr{M}_{R}$ with $z \notin M$. Since $T=R_{z}$ is a localization of $R$, we have $M T \in \mathscr{M}_{T}$ and $M T \cap R=M$ for such maximal right ideals $M$. Also, contraction of right ideals from $T$ to $R$, followed by extension back to $T$, fixes right ideals of $T$ (i.e., for right ideals $I$ of $T,(I \cap R) T=I)$. So,

$$
J_{1} T=(\bigcap M) T=(\bigcap(M T \cap R)) T=((\bigcap(M T)) \cap R) T=\bigcap(M T) ;
$$

that is, $J_{1} T \in \mathscr{L}(T)$. 
The mapping preserves order, since if $J \subseteq L$ for some $J, L \in \mathscr{L}(R)$ then $J_{0} \subseteq L_{0}$ and $J_{1} \subseteq L_{1}$. Next we show that it preserves proper order. If $J \subset L$, then either $J_{0} \subset L_{0}$ or $J_{1} \subset L_{1}$. In the former case, $\overline{J_{0}} \subset \overline{L_{0}}$ by the Noether Isomorphism Theorem. In the latter case, there exists a maximal right ideal $M$, not containing $z$, with $J_{1} \subseteq L_{1} \cap M \subset L_{1}$. Then $J_{1} T \subseteq\left(L_{1} \cap M\right) T \subset L_{1} T$ as in the proof of the previous lemma.

Since the mapping above is one-to-one, we have

$$
\begin{aligned}
\operatorname{Kmax}(R) & =\operatorname{dev} \mathscr{L}(R) \leq \operatorname{dev}(\mathscr{L}(R / P) \times \mathscr{L}(T)) \\
& =\sup \{\operatorname{dev} \mathscr{L}(R / P), \operatorname{dev} \mathscr{L}(T)\} \\
& =\sup \{\operatorname{Kmax}(R / P), \operatorname{Kmax}(T)\} . \quad
\end{aligned}
$$

Remarks. (1) Lemma 2 is false if $\mathrm{Kmax}$ is replaced by $\mathrm{Kdim}$, as can be seen in the proof of Lemma 3. Lenagan has a formula [L, Theorem 2.3] similar to Lemma 2 that says $\operatorname{Kdim}(R)=\sup \left\{\operatorname{Kdim}(R / z R)+1, \operatorname{Kdim}\left(R_{z}\right)\right\}$ and is not true for Kmax.

(2) The inequality in Lemma 2 can be strict, as can be seen by taking $R$ to be the local ring $k[x, z]_{(x, z)}$. In that case, we have $\operatorname{Kmax}(R)=\operatorname{Kmax}(R / z R)=$ $0<1=\operatorname{Kmax}\left(R_{z}\right)$.

\section{THE NILPOTENT CASE}

Let $\mathbf{g}$ be a nilpotent Lie algebra. We define a reducing quadruple of $\mathbf{g}$ relative to a Lie ideal $\mathbf{l}$ of $\mathbf{g}$ to be a quadruple $(x, y, z, \mathbf{h})$ such that

(1) $x, y, z \in \mathbf{l}$ and $z=[x, y]$ is a nonzero central element of $\mathbf{g}$,

(2) $\mathbf{h}$ is a Lie ideal of $\mathbf{l}$ with $[\mathbf{h}, y]=0$,

(3) $\mathbf{l}=\mathbf{h} \oplus k x$ as a vector space.

If $\mathbf{l}=\mathbf{g}$ then $(x, y, z, \mathbf{h})$ is a reducing quadruple following Dixmier [D, $\S 4.7 .7]$.

Recall that $\mathbf{g}$ being nilpotent of dimension $n$ means that there exist linearly independent elements $x_{1}, \ldots, x_{n} \in \mathbf{g}$ such that:

(1) $\mathbf{g}=k x_{1}+\cdots+k x_{n}$,

(2) $\mathbf{g}_{i}=k x_{1}+\cdots+k x_{i}$ is a Lie ideal of $\mathbf{g}$,

(3) $\left[\mathbf{g}, \mathbf{g}_{i}\right] \subseteq \mathbf{g}_{i-1}$ for each $i=1,2, \ldots, n$ (with $\mathbf{g}_{0}=(0)$ ).

Proposition. Let $\mathbf{g}$ be a finite-dimensional nonabelian nilpotent Lie algebra. Then there exists a reducing quadruple of $\mathbf{g}$ relative to $\mathbf{g}_{i}$ for some $i$.

Proof. With the notation as in the previous paragraph, we suppose $x_{1}, \ldots, x_{j-1}$ are central in $\mathbf{g}$, but $x_{j}$ is not. Choose the smallest $i$ greater than $j$ for which $\left[x_{i}, x_{j}\right] \neq 0$. Then letting $x=x_{i}, y=x_{j}, z=[x, y], \mathbf{h}=\mathbf{g}_{i-1}$, and $\mathbf{l}=\mathbf{g}_{i}$ gives a reducing quadruple of $\mathbf{g}$ relative to $\mathbf{l}$.

Remark. The 2-dimensional nonabelian Lie algebra does not satisfy the conclusion of this proposition; however, some completely solvable nonabelian Lie algebras do satisfy the conclusion of this proposition. In that case, it will be clear from the proof of the next lemma that Kmax of their enveloping algebras will be less than Kdim.

Lemma 3. Let $\mathbf{g}$ be a nonabelian nilpotent Lie algebra of dimension $n$. Then $\operatorname{Kmax}(U(\mathbf{g}))<n$. 
Proof. First we reduce to the case that $k$ is uncountable. We may do this since tensoring up to such a field satisfies the hypothesis of Corollary 3.

Consider $\mathbf{g}$ in the notation of the paragraph preceding the proposition. Let $\left(x_{i}, y, z, \mathbf{g}_{i-1}\right)$ be the reducing quadruple of $\mathbf{g}$ relative to $\mathbf{g}_{i}$ as in the proof of the proposition. Regarded as an element of $R=U(\mathbf{g}), z$ is central. Since $z \neq 0$ and $R$ is a domain, $\operatorname{Kmax}(R / z R) \leq \mathrm{Kdim}(R / z R)<n$.

Next we show $\operatorname{Kmax}\left(R_{z}\right) \leq \operatorname{Kdim}\left(R_{z}\right)<n$. Since $R$ can be written as an iterated Ore extension and $z$ is central,

$$
R_{z} \simeq U\left(\mathbf{g}_{i-1}\right)_{z}\left[x_{i}, \delta_{i} ; \ldots ; x_{n}, \delta_{n}\right],
$$

where the $\delta$ 's are determined by the appropriate Lie products.

Since $A=U\left(g_{i-1}\right)_{z}$ is a finitely generated algebra over an uncountable field, $z^{-1} y$ is central in $A$, and $\delta_{i}\left(z^{-1} y\right)=1$, it follows that $A\left[x_{i}, \delta_{i}\right]$ satisfies the hypothesis of [MR, Proposition 9.1.14]. This together with [MR, Lemma 6.5.3(ii)] gives

$$
\operatorname{Kdim}\left(U\left(\mathbf{g}_{i-1}\right)_{z}\left[x_{i}, \delta_{i}\right]\right)=\operatorname{Kdim}\left(U\left(\mathbf{g}_{i-1}\right)_{z}\right)=\operatorname{Kdim}\left(U\left(\mathbf{g}_{i-1}\right)\right)=i-1 .
$$

By [MR, 6.5.4], Kdim can only increase by one per Ore extension, so the bound stated in the lemma follows from Lemma 2, proving the lemma.

Remark. Suppose $\left(x_{i}, y, z, \mathbf{g}_{i}\right)$ and $\left(x_{i^{\prime}}, y^{\prime}, z^{\prime}, \mathbf{g}_{i^{\prime}}\right)$ are relative reducing quadruples of $\mathbf{g}$ arising as in the proof of the proposition and that $\left\{x_{i}, y, z, x_{i^{\prime}}, y^{\prime}, z^{\prime}\right\}$ is linearly independent over $k$. Then Lemma 2 can be applied to $R / z R$ and $R_{z}$ to show that $\operatorname{Kmax}(U(\mathbf{g})) \leq \mathrm{Kdim}(U(\mathbf{g}))-2$. In fact, this procedure yields the following generalization of the theorem.

Corollary 4. Suppose $\mathbf{g}_{1}, \ldots, \mathbf{g}_{r}$ are finite-dimensional nonabelian nilpotent Lie algebras, $\mathbf{g}=\mathbf{g}_{1} \oplus \cdots \oplus \mathbf{g}_{r}$, and $\operatorname{dim}(\mathbf{g})=n$. Then $\operatorname{Kmax}(U(\mathbf{g})) \leq n-r$, and consequently, $\operatorname{sr}(U(\mathbf{g})) \leq n-r+1$, with equality if the underlying field has sufficiently large transcendence degree over the field of rational numbers.

\section{ORe eXtensions AND SOlVABLE Lie ALgebras}

In this section we will study certain iterated Ore extensions over a field of arbitrary characteristic in order to get upper bounds on $\mathrm{Kmax}$ for those rings. This class of Ore extensions includes the enveloping algebras of some solvable Lie algebras, for example, the 2-dimensional nonabelian Lie algebra.

Suppose $k$ is a field and

$$
R \simeq k\left[x_{1}, \ldots, x_{i-1}\right]\left[x_{i}, \delta_{i} ; \ldots ; x_{n}, \delta_{n}\right],
$$

where the $x$ 's are indeterminates and the $\delta$ 's are derivations. Suppose further that there exists some $j<i$ with $\delta_{i}\left(x_{j}\right)=\alpha x_{t}$ for some $\alpha \in k^{*}, t<i$, and $x_{t}$ normal in $R$.

Lemma 4. Let $R$ be as above. Then $\operatorname{Kmax}(R)<n$.

Proof. Once again, we can tensor up to a suitable field extension of $k$ by Corollary 3. Hence we may assume that $k$ is algebraically closed. Since $z=x_{t}$ is normal in $R$, we can apply Lemma 2 . The factor $R / x_{t} R$ has Kmax less than $n$ as in the proof of Lemma 3.

Suppose $M$ is a maximal right ideal of $A=k\left[x_{1}, \ldots, x_{i-1}\right]_{x_{t}}$ and $\overline{\delta_{i}}$ is the unique extension of $\delta_{i}$ to $A$. By the closure of $k, x_{j}-\beta \in M$ for some 
$\beta \in k$. Then $\alpha x_{t}=\overline{\delta_{i}}\left(x_{j}-\beta\right) \in \overline{\delta_{i}}(M)$. Thus there are no $\overline{\delta_{i}}$-invariant maximal ideals of $A$. By $[\mathrm{MR}, \S 6.9], \operatorname{Kdim}(A)=\operatorname{Kdim}\left(A\left[x_{i}, \overline{\delta_{i}}\right]\right)$. Since

$$
R_{x_{t}} \simeq A\left[x_{i}, \overline{\delta_{i}} ; \ldots ; x_{n}, \overline{\delta_{n}}\right]
$$

and Krull dimension can only increase by one per Ore extension, $\operatorname{Kmax}\left(R_{x_{t}}\right) \leq$ $\operatorname{Kdim}\left(R_{x_{t}}\right)<n$. Applying Lemma 2 completes the proof of this lemma.

Applying the Bass Stable Range Theorem provides the following result and its corresponding applications.

Corollary 3. Let $\mathbf{g}$ be the 2-dimensional nonabelian Lie algebra over any field. Then $\operatorname{sr}(U(\mathbf{g}))=2$.

\section{REFERENCES}

[B] H. Bass, K-theory and stable algebra, Inst. Hautes Études Sci. Publ. Math. 22 (1964), 489544.

[D] J. Dixmier, Enveloping algebras, North-Holland Math. Library, vol. 14, North-Holland, Amsterdam, 1977.

[GR] P. Gabriel and R. Rentschler, Sur la dimension des annueaux et ensembles ordonnés, C. $R$. Acad. Sci. Paris 265 (1967), 712-715.

[L] T. Lenagan, Krill dimension and invertible ideals of Noetherian rings, Proc. Edinburgh Math. Soc. (2) 20 (1976), 81-86.

[MR] J. McConnell and J. Robson, Noncommutative Noetherian rings, Wiley-Interscience, Chichester, 1987.

[St1] J. Stafford, On the stable range of right Noetherian rings, Bull. London Math. Soc. 13 (1981), 39-41.

[St2] _ Absolute stable rank and quadratic forms over noncommutative rings, K-Theory 4 (1990), 121-130.

[Su] A. Suslin, Reciprocity laws and the stable rank of polynomial rings, Math. USSR Izv. 15 (1980), 589-623.

Department of Mathematics, Texas A \& I University, Campus Box 172, Kingsville, TEXAS 78363-8201

E-mail address: kfgt000@taivm1.bitnet 